\title{
ATRIBUTOS QUÍMICOS E FÍSICOS DE SOLOS CULTIVADOS COM BANANEIRA 'PRATA-ANÃ' (AAB), EM TRÊS NÍVEIS DE PRODUTIVIDADE, NO NORTE DE MINAS GERAIS ${ }^{1}$
}

\author{
JOSÉ TADEU ALVES DA SILVA², DILERMANDO DOURADO PACHECO ${ }^{2}$ ÉDIO LUIZ DA COSTA ${ }^{4}$
}

RESUMO - A bananeira, para alcançar alta produtividade, deve ser cultivada em locais com fatores climáticos favoráveis e que apresentam solos com boa estrutura físico-química. O presente trabalho tem o objetivo de avaliar os atributos químicos e físicos de solos cultivados com bananeira 'Prata-anã' em três níveis de produtividade. Foram selecionados 96 bananais explorados comercialmente com bananeiras da cultivar Prata-anã (AAB), no norte de Minas Gerais. Os bananais foram divididos em três níveis de produtividade: baixa produtividade $\quad\left(<25 \mathrm{tha}^{-1} \mathrm{ano}^{-1}\right)$, produtividade média $\left(25 \mathrm{a} 32 \mathrm{t} \mathrm{ha}^{-1}\right)$ e alta produtividade $\left(>32 \mathrm{t} \mathrm{ha}^{-1} \mathrm{ano}^{-1}\right)$. Em cada bananal selecionado, foram coletadas amostras de solo e realizadas análises químicas e físicas. Amostras de folhas de cada bananal foram coletadas em 20 plantas. Nas amostras foliares, foram determinados os teores de N, P, K, Ca, Mg, S, B, Cu, Fe, Mn e Zn. Verificou-se que os teores de Ca, K, P, matéria orgânico, os valores de condutividade elétrica, CTC, a porcentagem de saturação por bases (V\%) e as quantidades de argila e silte apresentaram-se significativamente maiores nos solos dos bananais com alta produtividade. Nos solos dos bananais com alta produtividade, as quantidades de $\mathrm{Ca}, \mathrm{K}$ e $\mathrm{Mg}$ corresponderam a $82 \%, 5 \%$ e $13 \%$ da saturação por bases, respectivamente. Não houve diferenças entre os teores foliares de nutrientes dos bananais nos três níveis de produtividade.

Termos para indexação: Musa spp., relação Ca: $\mathrm{K}: \mathrm{Mg}$, avaliação nutricional

\section{CHEMICALAND PHYSICAL PROPERTIES OF SOIL CULTIVATED WITH DWARF PRATA BANANA TREE (AAB), IN THREE LEVELS OF PRODUCTIVITY, IN THE NORTH OF MINAS GERAIS}

\begin{abstract}
In order to reach high productivity, banana crops must be cultivated in places with favorable climatic factors that present soil with good chemical and physical properties. The present work has the objective to evaluate the chemical and physical properties of soil cultivated with Prata dwarf banana crops (AAB) in three levels of productivity. A total of 96 commercial banana crops were selected in the North of Minas Gerais. The banana crops were divided in three levels of productivity: low productivity $(<$ $25 \mathrm{t} \mathrm{ha}^{-1}$ year $\mathrm{r}^{-1}$ ), average productivity ( 25 the $32 \mathrm{t} \mathrm{ha}^{-1}$ year $^{-1}$ ) and high productivity $\left(>32 \mathrm{tha}^{-1}\right.$ year $\left.{ }^{-1}\right)$. In each selected banana crop, soil samples were collected for chemical and physical analyses. Leaf samples of each banana crop were collected in 20 plants. In the foliares samples were determined the $\mathrm{N}, \mathrm{P}, \mathrm{K}, \mathrm{Ca}, \mathrm{Mg}, \mathrm{S}, \mathrm{B}, \mathrm{Cu}, \mathrm{Fe}, \mathrm{Mn}$ and $\mathrm{Zn}$ proportions. It was verified that the content of $\mathrm{Ca}, \mathrm{K}$, $\mathrm{P}$, organic matter, the values of electric conductivity, CTC, the percentage of saturation per bases (V\%), and the amount of clay and silte present were significantly greater in the banana crops of high productivity soil. It was verified that in the banana crops of high productivity soil the amounts of $\mathrm{Ca}, \mathrm{K}$ and $\mathrm{Mg}$ corresponded to $82 \%, 5 \%$ and $13 \%$ of the base saturation, respectively. There was no difference among the foliar contents of nutrients in the three banana crops levels of productivity.
\end{abstract}

Index terms: Musa spp., relation Ca:K:Mg, nutritional evaluation.

\section{INTRODUÇÃO}

Dentre os vários fatores ligados ao sistema de produção, a nutrição é de fundamental importância, pois, para alcançar alta produtividade econômica, é necessário que a bananeira esteja equilibrada nutricionalmente (Silva \& Carvalho, 2005).

Em trabalho realizado por Damatto Junior et al. (2006), os autores verificaram que os teores de nutrientes em folhas de bananeira 'Prata-anã' apresentaram a seguinte ordem de concentração na época do florescimento das plantas: $\mathrm{K}>\mathrm{N}>\mathrm{Ca}$ $>\mathrm{Mg}>\mathrm{S}>\mathrm{P}$.

O N é o nutriente responsável pelos aumentos do número de folhas e suas dimensões, pelos números de pencas e de frutos de banana, pela emissão e crescimento dos rebentos. O K encontra-se em alta quantidade na planta. Além de ser um nutriente importante na produção de frutos, aumenta a resistência destes ao transporte e melhora a sua qualidade (Silva et al., 1999).

A utilização de solos pouco férteis e a falta de manutenção de níveis adequados de nutrientes durante o ciclo da planta são os principais fatores da baixa produtividade da bananeira (MartinPrével,1985). Aliadas a isso, as elevadas quantidades de nutrientes retirados do solo durante os vários ciclos produtivos da cultura podem comprometer ainda mais o equilíbrio nutricional da planta (Borges et al., 1995; Silva et al., 1999).

No trabalho realizado por Teixeira et al. (2001), os autores verificaram que a adubação nitrogenada, com a utilização do nitrato de amônio em bananeira, determinou incrementos significativos na acidez do solo, diminuindo a saturação por bases

\footnotetext{
${ }^{1}$ (Trabalho 084-2006). Recebido em 20-06-2006. Aceito para publicação em 13-02-2007. Trabalho apresentado no XXX Congresso Brasileiro de Ciência do Solo. Financiado pela FAPEMIG

${ }^{2}$ Pesquisador da EPAMIG/CTNM, DSc. Solos e Nutrição de Plantas, Caixa Postal 12, Nova Porteirinha-MG, e-mail: josetadeu@epamig.br

${ }^{3}$ Pesquisador da EPAMIG/CTNM, DSc. Fitotecnia, Caixa Postal 12, Nova Porteirinha-MG, e-mail: dd_pacheco@epamig.br

${ }^{4}$ Pesquisador da EPAMIG/CTNM, DSc. Engenharia Agrícola, Caixa Postal 12, Nova Porteirinha-MG, e-mail: edio.costa@epamig.br
} 
e o teor de Mg trocável. Segundo esses autores, os efeitos do cultivo de bananeiras sobre alguns atributos químicos do solo indicaram a necessidade de monitoramento periódico da fertilidade, visando à manutenção de condições satisfatórias para a produção, especialmente quanto à acidez e aos teores de $\mathrm{K}$ e $\mathrm{Mg}$ trocáveis.

A bananeira é uma planta sensível ao desequilíbrio nutricional. Para elevar a produtividade desta cultura, é importante manter no solo o equilíbrio entre os nutrientes, evitando que ocorra consumo excessivo de um elemento induzindo deficiência de outro (Gutierrez, 1983).

O conhecimento das interações entre os principais nutrientes é importante no manejo da adubação da bananeira. $\mathrm{O}$ desbalanço entre $\mathrm{K} \mathrm{e} \mathrm{Mg}$ pode ocorrer com freqüência, em razão das elevadas quantidades de $\mathrm{K}$ aplicadas nos solos para atender às exigências da planta. A elevada relação $\mathrm{K} / \mathrm{Mg}$ pode favorecer o aparecimento do azul-da-bananeira, que se manifesta quando

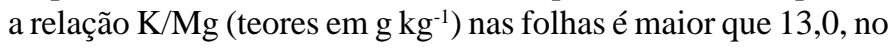
período de florescimento das plantas (Borges, 2004).

Delvaux (1995) relata que solos que apresentam relações $\mathrm{K} / \mathrm{Mg}$ entre 0,30 a 0,45 , encontram-se balanceados. Assim, relação superior a 0,6 indica excesso de $\mathrm{K}$, enquanto inferior a 0,2 mostra deficiência desse nutriente no solo. De acordo com este autor, teores de $\mathrm{K}$ no solo, que variam de 200 a $350 \mathrm{mg} \mathrm{dm}^{-3}$, são normalmente suficientes para o crescimento e produção da bananeira; entretanto, se o $\mathrm{Ca}$ e $\mathrm{Mg}$ estiverem presentes em quantidades elevadas, pode ocorrer deficiência de $\mathrm{K}$.

A relação $\mathrm{Ca} /(\mathrm{K}+\mathrm{Ca}+\mathrm{Mg})$ deve ficar em torno de $0,6 \mathrm{a}$ 0,8 , o que corresponde a uma proporção média de $70 \%$ de Ca. Assim, para o bom desenvolvimento da bananeira, as quantidades de K, Ca e Mg devem corresponder a 10\%, 60-70\% e 20-30\% da saturação por bases, respectivamente. Quando há deficiência de $\mathrm{K}$, a relação $\mathrm{K} / \mathrm{K}+\mathrm{Ca}+\mathrm{Mg}$ fica em 0,04 , enquanto com excesso, a relação é de 0,2 (Martin-Prével,1985).

Silva \& Carvalho (2004) verificaram que, em solos irrigados com águas calcárias, as quantidades de $\mathrm{K}, \mathrm{Ca}$ e $\mathrm{Mg}$ corresponderam a $5 \%, 84 \%$ e $11 \%$ da saturação por bases, respectivamente, enquanto os solos irrigados com águas nãocalcárias apresentaram quantidades de $\mathrm{K}, \mathrm{Ca}$ e Mg equivalentes a $7 \%, 75 \%$ e $18 \%$ da saturação por bases, respectivamente. Os autores verificaram que as bananeiras irrigadas com águas calcárias apresentaram produtividade média significativamente maior em relação àquelas irrigadas com águas não-calcárias, no período de um ciclo de avaliação de 100 bananais com idade entre dois a quatro anos.

De acordo com Souza et al. (1999), é importante para bananeira não somente os teores absolutos de $\mathrm{K}$, Ca e $\mathrm{Mg}$ no solo, mas, principalmente, o equilíbrio entre eles.

O presente trabalho tem o objetivo de avaliar os atributos químicos e físicos de solos cultivados com bananeira 'Prataanã', em três níveis de produtividade, no norte de Minas Gerais.

\section{MATERIAL E MÉTODOS}

As coletas dos dados foram realizadas entre julho de 2001 a fevereiro de 2004. Foram selecionados 96 bananais explorados comercialmente com bananeiras da cultivar Prata-anã (AAB), irrigados, com idade entre três e quatro anos, localizados no semi-árido do norte de Minas Gerais, nos municípios de Janaúba, Porteirinha, Nova Porteirinha, Jaíba, Matias Cardoso, Verdelândia e Capitão Enéas. Esses municípios estão situados entre os meridianos $41^{\circ} 44^{\prime} 35^{\prime \prime}$ e $45^{\circ} 27^{\prime} 33^{\prime \prime}$ de longitude a oeste e entre os paralelos $17^{\circ} 53^{\prime} 13^{\prime \prime}$ e $14^{\circ} 25^{\prime} 22^{\prime \prime}$ de latitude sul. Essa região está sob um clima do tipo Aw, segundo a classificação de Köppen (Moreira, 1985). As águas utilizadas nas irrigações dos bananais foram provenientes de rios e de poços artesianos.

No início do florescimento das bananeiras, em cada área selecionada, foram coletadas aleatoriamente amostras de solo com estruturas indeformadas, na profundidade de 0 a $5 \mathrm{~cm}$, para determinar a densidade do solo. Para a realização desta coleta, utilizou-se o trado de anéis (cilindro volumétrico). A densidade do solo foi determinada conforme descrito em Embrapa (1997). Amostras de solo na camada de 0 a $20 \mathrm{~cm}$ de profundidade foram coletadas, utilizando o trado tipo holandês. Cada amostra de solo foi composta de 20 amostras simples. Nestas amostras, foram determinados o pH, Ca, Mg, K, P, matéria orgânica (MO), condutividade elétrica (CE) e a granulometria , conforme Embrapa (1997).

As coletas das amostras simples de solo foram realizadas sempre em locais próximos às plantas. A $3^{\mathrm{a}}$ folha a partir do ápice foi coletada, retirando-se a parte central do limbo de cada folha, conforme Silva et al. (2002). A amostra de folha de cada bananal foi composta da coleta de material de 20 plantas. Nas amostras foliares, foram determinados os teores de $\mathrm{N}, \mathrm{P}, \mathrm{K}, \mathrm{Ca}, \mathrm{Mg}, \mathrm{S}, \mathrm{B}$, $\mathrm{Cu}, \mathrm{Fe}, \mathrm{Mn}$ e Zn, de acordo com métodos descritos por Malavolta et al. (1997). Cada planta, onde se coletou amostra de folha, foi marcada com tinta. Os cachos das plantas marcadas foram colhidos, despencados e pesados. A colheita dos cachos foi realizada quando os frutos apresentavam mudanças de tonalidades de verde-escuro para verde-claro. De acordo com o peso dos cachos, os bananais foram divididos em três níveis de produtividade: baixa produtividade $\left(<25 \mathrm{t} \mathrm{ha}^{-1} \mathrm{ano}^{-1}\right)$,

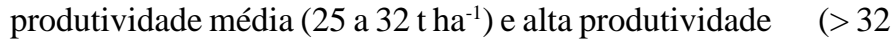
$\mathrm{t} \mathrm{ha}^{-1} \mathrm{ano}^{-1}$ ). Esses limites de produtividade foram estabelecidos considerando que, para a bananeira 'Prata-anã' cultivada no norte de Minas Gerais ser rentável, é necessário que sua produtividade média esteja próxima de $28 \mathrm{tha}^{-1} \mathrm{ano}^{-1}$. Os limites de $25 \mathrm{a} 32 \mathrm{tha}$ ${ }^{1}$ ano $^{-1}$ foram estabelecidos para se ter um intervalo de segurança.

As análises de variância foram realizadas para os atributos químicos e físicos do solo e para os teores de nutrientes foliares. Nas análises de variância, utilizou-se de um delineamento inteiramente casualisado, com três tratamentos (produtividade baixa, média e alta) e 32 repetições, totalizando-se 96 parcelas. Para os testes de média, aplicou-se o teste de Tukey, a 5\% de probabilidade.

\section{RESULTADOS E DISCUSSÃO}

Verificou-se que os teores de $\mathrm{P}, \mathrm{K}, \mathrm{Ca}$ e MO, os valores de CE, CTC e a porcentagem de saturação por bases (V\%) se apresentaram significativamente maiores nos bananais de alta produtividade em relação aos de baixa produtividade (Tabela 1). 
As quantidades de $\mathrm{Ca}, \mathrm{K}$ e $\mathrm{Mg}$ corresponderam a $80 \%$, $5 \%$ e $15 \%$ da saturação por bases, respectivamente, nos solos dos bananais de baixa produtividade. Nos solos dos bananais de média produtividade, corresponderam a $79 \%, 7 \%$ e $14 \%$ e, nos solos dos bananais de alta produtividade, esses valores corresponderam a $82 \%, 5 \%$ e $13 \%$ da saturação por bases, respectivamente (Figura 1). As quantidades de $\mathrm{Ca}, \mathrm{K}$ e $\mathrm{Mg}$, que corresponderam à percentagem de saturação por bases, não apresentaram diferenças entre os solos dos três níveis de produtividade, pelo teste de Tukey, a $5 \%$ de probablidade. Segundo Martin-Prével (1985), para o bom desenvolvimento da bananeira, as quantidades de $\mathrm{Ca}, \mathrm{K}$ e $\mathrm{Mg}$ devem corresponder a $60-70 \%, 10 \%$ e $20-30 \%$ da saturação por bases, respectivamente. Portanto, a relação entre $\mathrm{Ca}: \mathrm{K}: \mathrm{Mg}$ obtida em solos cultivados com bananeira 'Prata-anã' com alta produtividade, no norte de Minas, apresentou pequena diferença da preconizada por MartinPrével (1985).

Silva et al. (2002) verificaram correlação positiva entre a produção de banana e o teor de Ca no solo. Segundo esses autores, a presença de elevadas quantidades de Ca no solo é muito importante para a bananeira alcançar alta produtividade. Entretanto, o $\mathrm{K}$ e $\mathrm{Mg}$ devem estar em níveis adequados para evitar que ocorra desequilíbrio entre esses cátions. Segundo Furtini Neto et al. (2001), o Ca é o nutriente mais importante para o crescimento de raízes, favorecendo a absorção de água e nutrientes pelas plantas.

Os solos dos bananais com alta produtividade apresentaram maiores quantidades de MO, argila e silte, e maior valor de CTC (Tabelas 1 e 2). A presença da MO melhora a qualidade das propriedades químicas e físicas dos solos. A CTC do solo apresenta correlação positiva com a quantidade e a qualidade da MO, argila e silte. Solos com altos valores desses atributos apresentam maior capacidade de reter água e nutrientes, proporcionando condições favoráveis para que a planta alcance alta produtividade (Silva et al., 1999).

Nos solos com bananais de baixa produtividade, observaram-se maiores quantidades de areia (Tabela 2). Silva et al. (2002) verificaram correlação negativa entre a produção da bananeira 'Prata-anã'e o teor de areia do solo. De acordo com
Silva et al. (1999), deve-se evitar o cultivo de bananeiras em solos arenosos em razão de apresentarem baixo teor de matéria orgânica, baixa retenção de umidade e nutrientes.

$\mathrm{O}$ valor da $\mathrm{CE}$ apresentou-se maior nos solos dos bananais de alta e média produtividades (Tabela 1). De acordo com Silva et al. (2002), a bananeira apresenta bom desenvolvimento em solo com CE de até $1 \mathrm{dS} \mathrm{m}^{-1}$; acima desse valor, ocorre comprometimento da produção. $\mathrm{O}$ aumento da $\mathrm{CE}$ até o limite tolerado pela bananeira favorece a floculação da argila e, conseqüentemente, a redução da densidade do solo, proporcionando melhor desenvolvimento das raízes das plantas, aumentando o potencial dessas para absorção de água e nutrientes. Não ocorreram diferenças estatísticas entre as densidades dos solos dos bananais, nos três níveis de produtividade (Tabela 2). Souza et al. (1999) determinaram os valores da densidade de quatro diferentes solos (NQ, LVA, LV e NF) cultivados com bananeira no norte de Minas Gerais. Verificaram que, de forma geral, estes solos apresentaram valores de densidade elevados (1,29 a 1,69 $\left.\mathrm{kg} \mathrm{dm}^{-3}\right)$.

Quanto aos teores foliares de nutrientes, nos três níveis de produtividades, não houve diferenças significativas (Tabela 3). De acordo com a interpretação baseada em Alvarez V. et al. (1999), os solos dos bananais de alta, média e baixa produtividades apresentaram bons níveis de fertilidade (Tabela 1). Esse fato demonstra que não foi por deficiência nutricional a baixa produtividade $\left(<25 \mathrm{tha}^{-1} \mathrm{ano}^{-1}\right)$ dos bananais. Os teores de $\mathrm{Ca}, \mathrm{K}$ e $\mathrm{P}$ nos solos dos bananais de baixa produtividade (Tabela 1), mesmo apresentando valores significativamente menores em relação àqueles dos bananais de alta produtividade, estão acima dos valores considerados adequados para obtenção de alta produtividade, conforme Silva et al. (2002). Portanto, não houve limitações de nutrientes no solo para as plantas; por isso, os teores foliares de nutrientes apresentaram-se semelhantes nos três níveis de produtividade, confirmando que o motivo da baixa produtividade $\left(<25 \mathrm{t} \mathrm{ha}^{-1} \mathrm{ano}^{-1}\right)$ dos bananais não foi por deficiência nutricional. Silva \& Carvalho (2005) também detectaram este fato na avaliação do estado nutricional, utilizando o método DRIS para bananeira 'Prata-anã', cultivada no norte de Minas.

TABELA 1 - Valores médios do pH, P, K, Ca, Mg, CTC, condutividade elétrica (CE), matéria orgânica (MO) e saturação por bases (V) de solos cultivados com bananeira 'Prata- anã', em três níveis de produtividade, no norte de Minas Gerais, 2005.

\begin{tabular}{lcccccccccc}
\hline Fontes de Variação & $\mathrm{pH}$ & $\mathrm{P}^{\mathrm{I}}$ & $\mathrm{K}^{\mathrm{l}}$ & $\mathrm{Ca}$ & $\mathrm{Mg}$ & $\mathrm{CTC}$ & $\mathrm{CE}$ & $\mathrm{MO}$ & $\mathrm{V}$ & $\mathrm{K}+\mathrm{Ca}+\mathrm{Mg}$ \\
\hline & & $\mathrm{mg} \mathrm{dm}^{-3}$ & $--------------\mathrm{cmol}_{\mathrm{c}} \mathrm{dm}^{-3}-------------$ & $\mathrm{dS} \mathrm{m}{ }^{-1}$ & $\mathrm{dag} \mathrm{kg}^{-1}$ & $\%$ & $\mathrm{cmol}_{\mathrm{c}} \mathrm{dm}^{-3}$ \\
\hline Alta produtividade & $6,6 \mathrm{a}$ & $39,2 \mathrm{a}$ & $0,49 \mathrm{a}$ & $7,4 \mathrm{a}$ & $1,2 \mathrm{a}$ & $10,9 \mathrm{a}$ & $0,51 \mathrm{a}$ & $2,7 \mathrm{a}$ & $83 \mathrm{a}$ & 9,1 \\
Produtividade média & $6,2 \mathrm{a}$ & $22,5 \mathrm{~b}$ & $0,51 \mathrm{a}$ & $5,8 \mathrm{ab}$ & $1,0 \mathrm{a}$ & $9,5 \mathrm{ab}$ & $0,54 \mathrm{a}$ & $2,2 \mathrm{~b}$ & $79 \mathrm{ab}$ & 7,3 \\
Baixa Produtividade & $6,2 \mathrm{a}$ & $23,0 \mathrm{~b}$ & $0,32 \mathrm{~b}$ & $4,9 \mathrm{~b}$ & $0,9 \mathrm{a}$ & $8,2 \mathrm{~b}$ & $0,39 \mathrm{~b}$ & $1,8 \mathrm{~b}$ & $74 \mathrm{~b}$ & 6,1 \\
\hline Média & 6,3 & 28,2 & 0,44 & 6,0 & 1,0 & 9,5 & 0,48 & 2,2 & 79 & 7,5 \\
\hline C.V \% & 14,5 & 75,4 & 61,1 & 48,2 & 56,3 & 33,1 & 64,4 & 40,1 & 15,4 & 31,1 \\
\hline Médias seguidas da mesma & letra, entre linhas, não diferem entre si, pelo & teste & de Tukey, a 5\% de probalidade. ${ }^{1}$ Extrator Melich-1
\end{tabular}

Médias seguidas da mesma letra, entre linhas, não diferem entre si, pelo teste de Tukey, a 5\% de probalidade. ${ }^{1}$ Extrator Melich-1 
TABELA 2 - Valores médios dos teores de argila, silte, areia e de densidade de solos cultivados com bananeira 'Prata-anã', em três níveis de produtividade, no norte de Minas Gerais, 2005.

\begin{tabular}{|c|c|c|c|c|}
\hline Fontes de Variação & Argila & Silte & Areia & Densidade \\
\hline & --------------- & ag kg ${ }^{-1}=$ & ---------- & $\left(\mathrm{kg} \mathrm{dm}^{-3}\right)$ \\
\hline Alta produtividade & $31,4 \mathrm{a}$ & $26,1 \mathrm{a}$ & $42,5 \mathrm{~b}$ & $1,5 \mathrm{a}$ \\
\hline Produtividade média & $31,4 \mathrm{a}$ & $21,8 \mathrm{ab}$ & $46,8 \mathrm{ab}$ & $1,7 \mathrm{a}$ \\
\hline Baixa Produtividade & $26,0 \mathrm{~b}$ & $20,1 \mathrm{~b}$ & $53,9 \mathrm{a}$ & $1,7 \mathrm{a}$ \\
\hline Média & 29,6 & 22,7 & 47,7 & 1,6 \\
\hline C.V \% & 41,8 & 45,5 & 41,7 & 13,6 \\
\hline
\end{tabular}

Médias seguidas da mesma letra, entre linhas, não diferem entre si, pelo teste de Tukey, a 5\% de probabilidade.

TABELA 3 - Valores médios dos teores de nutrientes foliares de bananeira 'Prata-anã', em três níveis de produtividade, no norte de Minas Gerais, 2005.

\begin{tabular}{|c|c|c|c|c|c|c|c|c|c|c|c|}
\hline $\begin{array}{l}\text { Fontes de } \\
\text { Variação }\end{array}$ & $\mathrm{N}$ & $\mathrm{P}$ & $\mathrm{K}$ & $\mathrm{Ca}$ & $\mathrm{Mg}$ & $\mathrm{S}$ & B & $\mathrm{Cu}$ & $\mathrm{Fe}$ & $\mathrm{Mn}$ & $\mathrm{Zn}$ \\
\hline & \multicolumn{6}{|c|}{ - } & \multicolumn{5}{|c|}{ - } \\
\hline $\begin{array}{l}\text { Alta } \\
\text { produtividade }\end{array}$ & $26,1 \mathrm{a}$ & $1,6 \mathrm{a}$ & $32,3 \mathrm{a}$ & $6,1 \mathrm{a}$ & $2,9 \mathrm{a}$ & $2,1 \mathrm{a}$ & $27,9 \mathrm{a}$ & $5,5 \mathrm{a}$ & $115,0 \mathrm{a}$ & $458,8 \mathrm{a}$ & $17,2 \mathrm{a}$ \\
\hline $\begin{array}{l}\text { Produtividade } \\
\text { média }\end{array}$ & $26,2 \mathrm{a}$ & $1,6 \mathrm{a}$ & $30,3 \mathrm{a}$ & $6,0 \mathrm{a}$ & $3,3 \mathrm{a}$ & $1,9 \mathrm{a}$ & $28,5 \mathrm{a}$ & $5,8 \mathrm{a}$ & $115,1 \mathrm{a}$ & $409,2 \mathrm{a}$ & $15,7 \mathrm{a}$ \\
\hline Baixa & $26,0 \mathrm{a}$ & $1,70 \mathrm{a}$ & $30,2 \mathrm{a}$ & $5,7 \mathrm{a}$ & $3,3 \mathrm{a}$ & $2,1 \mathrm{a}$ & $28,2 \mathrm{a}$ & $6,2 \mathrm{a}$ & $124,1 \mathrm{a}$ & $504,8 \mathrm{a}$ & $15,4 \mathrm{a}$ \\
\hline Produtividade & & & & & & & & & & & \\
\hline Média & 26,1 & 1,6 & 30,9 & 5,9 & 3,2 & 2,0 & 28,2 & 5,8 & 118,1 & 457,6 & 16,1 \\
\hline C.V \% & 11,2 & 12,3 & 14,1 & 22,6 & 22,7 & 20,8 & 10,7 & 55,1 & 31,5 & 85,5 & 24,1 \\
\hline
\end{tabular}

Médias seguidas da mesma letra, entre linhas, não diferem entre si, pelo teste de Tukey, a 5\% de probabilidade.

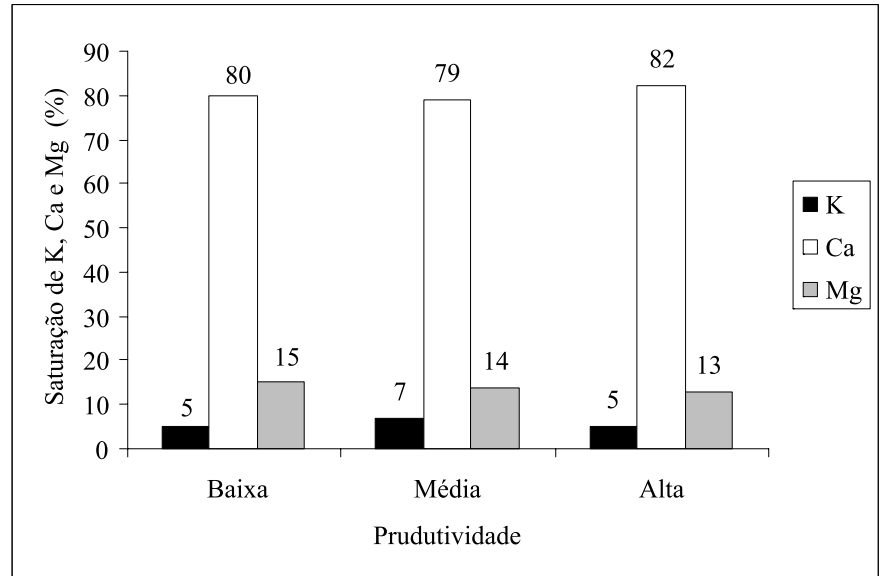

FIGURA 1 - Porcentagem da saturação do solo por $\mathrm{Ca}, \mathrm{K}$ e $\mathrm{Mg}$ em relação às bases $(\mathrm{Ca} / \mathrm{Ca}+\mathrm{K}+\mathrm{Mg}, \mathrm{K} / \mathrm{Ca}+\mathrm{K}$ $+\mathrm{Mg}$, e $\mathrm{Mg} / \mathrm{Ca}+\mathrm{K}+\mathrm{Mg}$ ).
Os fatores não-nutricionais que possivelmente influenciaram na produtividade das bananeiras, foram a granulometria do solo e o manejo da cultura, tais como controle de pragas e doenças, manejo da irrigação e desbrota. Quanto à granulometria, os solos das bananeiras de baixa produtividade apresentaram teor de areia significativamente maior em relação aos das bananeiras de alta produtividade (Tabela 2). Solos com maior teor de areia apresentam menor potencial produtivo (Silva et al., 1999; Silva et al., 2002).

\section{CONCLUSÕES}

Os solos dos bananais de alta produtividade apresentaram maiores teores de $\mathrm{P}, \mathrm{K}, \mathrm{Ca}$ e matéria orgânica, maiores valores de condutividade elétrica, CTC e saturação por bases em relação aos solos dos bananais de baixa produtividades.Não ocorreram diferenças entre os teores foliares de nutrientes dos bananais de alta, média e baixa produtividades. 


\section{REFERÊNCIAS}

ALVAREZ V., V. H.; NOVAIS, R. F de; BARROS, N. F.de; CANTARUTTI, R. B.; LOPES, A. S. Interpretação dos resultados das análises de solos. In: RIBEIRO, A. C.; GUIMARÃES, P. T. G.; ALVAREZ, V., H., Recomendações para o uso de corretivos e fertilizantes em Minas Gerais. $5^{\text {a }}$ aproximação,Viçosa, 1999, p. 25 -32.

BORGES, A.L.; OLIVEIRA, A.M.G.; SOUZA, L. da S. Solos, nutrição e adubação da bananeira. Cruz das Almas : EmbrapaCNPMF, 1995. 44 p. (Embrapa-CNPMF. Circular Técnica, 22).

BORGES, A. L. Interação entre nutrientes em bananeira. Cruz das Almas: Embrapa-CNPMF, 2004. 2 p. (Embrapa-CNPMF. Banana em Foco, 55).

DAMATTO JUNIOR, E. R.; VILLAS BÔAS, R. L.; LEONEL, S.; FERNANDES, D. M. Avaliação nutricional em folhas de bananeira 'Prata anã' adubadas com composto orgânico. Revista Brasileira de Fruticultura, Jaboticabal, v.28, n.1, p.109-112, 2006.

DELVAUX, B. Soil. In: GOWEN, S. Bananas and plantains. New York: Chapman \& Hall, 1995. p. 230-257.

EMBRAPA.Centro Nacional de Pesquisa de solos. Manual de métodos de análise de solo. 2.ed. revisado e atualizado. Rio de Janeiro: Centro Nacional de Pesquisa de Solos, 1997. 212p.

FURTINI NETO, A. E.; VALE, F. R. do; RESENDE, A. V. de; GUILHERME, L. R. G.; GUEDES, G. A. A. Fertilidade do solo. Lavras: UFLA/FAEPE, 2001, 252p.

GUTIERREZ, C. A. L. Diagnóstico del estado nutricional de plantaciones bananeras. Asbana: v. 6, n. 19, p.13-18, 1983.

MALAVOLTA, E.; VITTI, G. C.; OLIVEIRA, S. A. Avaliação do estado nutricional das plantas: princípios e aplicações. 2. ed. Piracicaba: POTAFOS, 1997. 201 p.

MARTIN-PRÉVEL, P. Exigências nutricionais da bananicultura. In: SIMPÓSIO BRASILEIRO SOBRE BANANICULTURA, 1, 1984, Jaboticabal. Anais... Jaboticabal. UNESP/FCAV, 1985, p. 118-134.

MOREIRA, I. A. G. Geografia Geral e do Brasil. São Paulo, Moderna, 1985. 230p.

SILVA, J. T. A. ; BORGES A. L., MALBURG, J. L. SOLOS, ADUBAÇÃO ENUTRIÇÃO DA BANANEIRA. Informe agropecuário, Belo Horizonte, v. 20, n. 196, p. 21-36, 1999.

SILVA, J. T. A.; BORGES, A. L.; DIAS, M. S. C.; COSTA, E. L.; PRUDÊNCIO, J. M. Diagnóstico nutricional da bananeira Prata-anã para o Norte de Minas. Belo Horizonte: EPAMIG, 2002, 16 p. (Boletim Técnico, 70).

SILVA, J. T. A.; CARVALHO, J. G. de. Propriedades do solo, estado nutricional e produtividade de bananeiras 'Prata Anã'irrigadas com águas calcárias. Ciência e Agrotecnologia, Lavras, v. 28, n. 2, p. 334-340, 2004.

SILVA, J. T. A.; CARVALHO, J. G. . Avaliação nutricional de bananeira Prata-Anã (AAB), sob irrigação no semi-árido do norte de Minas Gerais, pelo método Dris. Ciência e Agrotecnologia, Lavras-MG, v. 29, n. 4, p. 731-739, 2005.
SOUZA, L.; SILVA; BORGES, L. A.; SILVA, J. T. A. da. Características físicas e químicas de solos cultivados com bananeira, sob irrigação, na região norte de Minas Gerais. Cruz das Almas-BA: Embrapa Mandioca e Fruticultura, 1999, 24p. (Boletim de Pesquisa, 14).

TEIXEIRA, L. A. J.; NATALE, W.; RUGGIERO. Alteração em alguns atributos químicos do solo decorrentes da irrigação e adubação nitrogenda e potássica em bananeira após dois ciclos de cultivo. Revista Brasileira de Fruticultura, Jaboticabal, v.23, p. 684-689, 2001. 